\title{
Disorder and transport in cuprates: weak localization and magnetic contributions
}

\author{
F.Rullier-Albenque ${ }^{1}$, H. Alloul ${ }^{2}$, R. Tourbot ${ }^{1}$ \\ ${ }^{1}$ SPEC, Orme des Merisiers, CEA, 91191 Gif sur Yvette, France \\ ${ }^{2}$ Laboratoire de Physique des Solides, UMR 8502, Université \\ Paris-Sud, 91405 Orsay, France
}

(November 2, 2018)

\begin{abstract}
We report resistivity measurements in underdoped $\mathrm{YBa}_{2} \mathrm{Cu}_{3} \mathrm{O}_{6.6}$ and overdoped $\mathrm{Tl}_{2} \mathrm{Ba}_{2} \mathrm{CuO}_{6+x}$ single crystals in which the concentration of defects in the $\mathrm{CuO}_{2}$ planes is controlled by electron irradiation. Low $T$ upturns of the resistivity are observed in both cases for large defect content. In the $\mathrm{Tl}$ compound the decrease of conductivity scales as expected from weak localization theory. On the contrary in $\mathrm{YBa}_{2} \mathrm{Cu}_{3} \mathrm{O}_{6.6}$ the much larger low $\mathrm{T}$ contribution to the resistivity is proportional to the defect content and might then be associated to a Kondo like spin flip scattering term. This would be consistent with the results on the magnetic properties induced by spinless defects.
\end{abstract}

The origin of the strange metallic properties of the cuprates, among which the transport properties, is still the key feature to be resolved to progress in the understanding of the physics of these $2 \mathrm{D}$ correlated electron systems. As the pseudogap is evidenced both in magnetic and transport properties, some interplay between spin and charge degrees of freedom is required. This interplay can be studied as well from the influence of defects and impurities on the transport properties. Point defects such as $\mathrm{Zn}$ impurities induce a strong depression of $T_{c}$ as expected for d-wave superconductivity. This is often considered to result from pure potential (unitary?) scattering [1]. However it has been shown that such spinless defects induce a local moment in their vicinity [5,6]. One can therefore wonder whether these magnetic degrees of freedom play a role in the charge scattering, and induce for instance a (Kondo like) spin flip contribution to the resistivity.

In presence of $\mathrm{Zn}$ impurities, low $T$ upturns of the resistivity with a $\ln T$ variation have been observed in $\mathrm{La}_{2} \mathrm{Sr}_{2} \mathrm{CuO}_{4}$ (LSCO) and $\mathrm{YBa}_{2} \mathrm{Cu}_{3} \mathrm{O}_{7-\delta}$ (YBCO) [7, 8 . However these observations have required large $\mathrm{Zn}$ contents or low hole doping in order to suppress superconductivity. Data were then limited to large values of the $2 \mathrm{D}$ resistivity which approach or exceed $\approx 5 k \Omega / \square$ for which deviations from weak localization predictions start to be observed in 2D systems [9, 10]. These experimental constraints have up to now prevented any thorough study either of the impurity contributions to the scattering, or of the applicability of 2D weak localization theory [12].

Recent accurate NMR measurements in the case of $\mathrm{Li}$ impurities, which behave similarly to Zn, have evidenced a Kondo like variation of the induced local moment susceptibility with a Kondo temperature $T_{K}$ which increases with hole doping [6]. One might expect that magnetic contributions to the transport properties should then differ markedly in underdoped and overdoped systems while localization effects should always be present.

In this work, we address both questions for the first time by comparing underdoped and overdoped cuprates. We have used electron irradiation to introduce in-plane point defects which behave similarly to $\mathrm{Zn}$ substitutions in YBCO [13,4]. As measurements could be performed on a single sample, the accuracy on the defect contribution to the resistivity was high enough to study thoroughly its variation with defect content and temperature, even when $T_{c}$ is still high. In the heavily overdoped $\mathrm{Tl}_{2} \mathrm{Ba}_{2} \mathrm{CuO}_{6+x}(\mathrm{Tl} 2201)$, we find here that $T_{c}$ can be suppressed while the low $T$ resistance is still below the strong localization limit. This enables us to demonstrate that weak localization theory applies and that defect scattering is purely elastic in this system. We shall show that this approach fails in underdoped $\mathrm{YBCO}_{6.6}$. The large magnitude of the low $T$ upturns of the resistivity and its variation with defect content rather indicates the occurence of a Kondo like inelastic spin-flip scattering. The difference between these two systems will be found consistent with the observed doping dependence of $T_{K}$.

We have taken data on the underdoped $\mathrm{YBCO}_{6.6}$ single crystal, which has already been used in ref [4]. It displayed the usual pseudo gap resistivity temperature dependence with an initial $T_{c}$ of $60 \mathrm{~K}$. The highly overdoped Tl2201 sample prepared similarly to those of ref 柾, with an initial $T_{c}$ of $30 \mathrm{~K}$, exhibited a power law dependence of $\rho(T)$ characteristic of the overdoped state [14]. The samples have been irradiated with $2.5 \mathrm{MeV}$ electrons ${ }^{(*)}$ in liquid hydrogen to allow high electron flux without sample heating. After a short time defect annealing $(\approx$ 10 minutes) either at $T_{a}=100 \mathrm{~K}, 150 \mathrm{~K}$ or $300 \mathrm{~K}$, resistivity measurements were performed between $20 \mathrm{~K}$ and $T_{a}$. In such conditions the resistivity versus $T$ curves plotted in Fig.1 were found absolutely reversible, indicating the absence of further evolution of the defect content. The high fluences required to reach large enough resistivities led us to perform several independent irradiation runs. This forced us to remove the probe from the irradiation facility and to let the samples stay at room $T$ for long time (typically a few months) after each run, which reduced subsequently the concentration of defects [4. The resistivity curves after annealing at $300 \mathrm{~K}$ are quite identical to those observed in situ for lower irradiation fluences. This indicates that the measured resistivity 
is only sensitive to the concentration of in-plane defects. After the last run the samples were removed from the irradiation probe and ex-situ measurements were then performed down to lower $T(1.7 \mathrm{~K})$ in a different set-up in which magnetoresistance data could be taken.

As can be seen in Fig.1 the high $T$ parts of the resistivity curves are nearly parallel to each other in $\mathrm{YBCO}_{6.6}$, i.e. Mathiessen's rule extends to large irradiation fluences [4, which implies also that the hole doping of the $\mathrm{CuO}_{2}$ planes is not significantly modified. Conversely the variation with $T$ of the resistivity of the Tl2201 sample increases with defect content showing that in this case the hole doping increases slightly [15]. In both compounds low $T$ upturns of the resistivity are observed and increase with defect content. These upturns appear at higher $T$ for $\mathrm{YBCO}_{6.6}$, and correspond to larger contributions to the $2 \mathrm{D}$ in-plane resistance $R_{2 D}$ than for Tl2201 [16].

To see this effect more quantitatively, we have reported in Fig. $2 \delta R_{2 D}$, the low $\mathrm{T}$ increase of $R_{2 D}$ obtained in these two compounds for comparable residual resistances $R_{2 D}^{*} \simeq 4.8 \mathrm{k} \Omega / \square$. In order to evaluate $\delta R_{2 D}$ the resistivity of the non irradiated compound had to be extrapolated below $T_{c}$. For pure $\mathrm{YBCO}_{6.6}$, two different empirical forms $R_{2 D}^{0}(T)=a+b T+c T^{3}$ and $R_{2 D}^{0}(T)=a+b T^{p}$ have been used to fit the data between $80 \mathrm{~K}$ and $150 \mathrm{~K}$. This lower $\mathrm{T}$ limit has been taken to avoid the $T$ range for which superconducting fluctuations contribute to the resistivity. Although their extrapolations differ significantly at low $T$, the magnitude and $T$ dependence obtained for $\delta R_{2 D}$ are quite identical in the limited experimental range above $T_{c}$. For Tl 2201, superconductivity has been suppressed for this fluence and the low $T$ extrapolation might be more critical than for $\mathrm{YBCO}_{6.6}$. To take into account the slight change of hole doping we have then fitted the high $T$ part of each resistivity curve with $R_{2 D}^{0}(T)=a+b T^{p}$. To test the validity of this procedure, two extreme cases have been considered. The fits were performed either between $70 \mathrm{~K}$ and $150 \mathrm{~K}$, or between $150 \mathrm{~K}$ and $300 \mathrm{~K}$. Although $\delta R_{2 D}$ is naturally found to depart from zero at a slightly higher $T$ in the latter case, the overall variation and particularly the $\ln T$ dependence found at low $T$ is not sensitive to the function chosen for $R_{2 D}^{0}(T)$. It is clear in Fig.2 that, whatever the fitting procedure, $\delta R_{2 D}$ is larger and has a steeper $T$ variation in $\mathrm{YBCO}_{6.6}$ than in the $\mathrm{Tl}$ compound.

We can at this stage compare these data with the expectations of the weak $2 \mathrm{D}$ localization theory, which can apply as long as the elastic mean free path $l$ verifies $k_{F} l>1$ (where $k_{F}$ is the Fermi wave vector), that is for $R_{2 D}<<26 k \Omega / \square$. The weak localization contributions are expected to become sizable at temperatures for which the elastic scattering rate $1 / \tau$ becomes much larger than the inelastic scattering rate $1 / \tau_{i}$. Assuming that the in-plane defects only contribute to the elastic scattering, the ratio $\tau_{i} / \tau$ can be estimated from the data, if the inelastic rate is associated to the $T$ dependent contribution to $R_{2 D}$ of the starting pure material. For instance, for the residual resistivity $R_{2 D}^{*} \simeq 4.8 \mathrm{k} \Omega / \square$ of Fig. $2, \tau_{i} / \tau$ $\approx 10-12$ and $\approx 7$ respectively for $\mathrm{Tl} 2201$ and $\mathrm{YBCO}_{6.6}$ at $100 \mathrm{~K}$ ( at $50 \mathrm{~K}$ the deduced value would be $\tau_{i} / \tau \approx 40$ for the two systems). Therefore, within the above assumptions, weak localization conditions are fulfilled even at such high $T$. Let us recall that the theoretical correction to the $2 \mathrm{D}$ conductance has a universal behaviour at low $T$ given by

$$
\Delta \sigma=\frac{\alpha e^{2}}{2 \pi^{2} \hbar} p \ln \left(T / T_{0}\right)
$$

where $p$ is the exponent of the $T$ dependence of the inelastic scattering rate $1 / \tau_{i} \propto T^{p}$, and $\alpha=1$ in the absence of electronic interactions, in good agreement with experimental observations 17. 20 .

We have plotted in Fig.3a the value of $\Delta \sigma=$ $\left(R_{2 D}\right)^{-1}-\left(R_{2 D}^{0}\right)^{-1}$ for the $\mathrm{Tl}$ compound. For the in-situ measurements the data are limited to $20 \mathrm{~K}$, while they could be pursued down to $3 \mathrm{~K}$ for the final state of the sample. In this case a good fit with a $\ln T$ dependence extends over a decade. One can notice in Fig.3a that the curves for the other defect contents are parallel to each other at $20 \mathrm{~K}$, with a slope identical to that found for the lower $T$ run, within experimental accuracy. Finally at a given $T$ the magnitude of $\Delta \sigma$ increases with defect concentration, as the upturn in the resistivity curve occurs at higher $\mathrm{T}$ as can be seen in Fig.1a. These qualitative observations are more striking in Fig. $3 \mathrm{~b}$ where we have performed the scaling of these curves in $\ln \left(T / T_{0}\right)$. Here $T_{0}$ is found to increase progressively from $70 \mathrm{~K}$ to $120 \mathrm{~K}$ with defect content. The actual slope obtained experimentally below $30 \mathrm{~K}$ yields the numerical value $\alpha p=1.3$, when compared to (11). The value of $p$ can be independently estimated from the $T$ dependence of $R_{2 D}^{0}(T)$ and varies from 1.7 to 2.3 . This would correspond to $\alpha \simeq 0.7$. These results provide a strong experimental evidence that $2 \mathrm{D}$ weak localization theory explains the Tl2201 data.

Transverse magnetoresistance measurements performed up to $8 \mathrm{~T}$ after the last irradiation provide an independent evidence which confirms this analysis. It indeed exhibits a negative contribution which decreases with increasing $\mathrm{T}$ as expected for weak localization. The field variation was accurate enough at low $T$ to allow an analysis along the lines of ref. [18], which allowed to deduce $\alpha \simeq 0.5$ and $\tau_{i} / \tau \simeq 60$ at $4.2 \mathrm{~K}$. While the value of $\alpha$ is in good agreement with the $T$ dependence of $R_{2 D}$, the value of $\tau_{i} / \tau$ is smaller than that which would be obtained from the extrapolation of the $T$ dependent part of the resistivity of the pure sample. Such a trend is quite common, as $\tau_{i}$ is usually found to flatten at low $T$, at values smaller, even in order of magnitude, than expected from the pure systems estimates $17,19,21$. Further experiments are needed to determine whether interaction effects contribute to $\Delta \sigma 22$.

Let us consider now the data for the underdoped compound $\mathrm{YBCO}_{6.6}$, for which we have seen that $\tau_{i} / \tau$ is weaker or similar, whereas $\delta R_{2 D}$ is larger than for the 
overdoped compound. In this case superconductivity prohibits to investigate the low $T$ range, and weak localization contributions are not expected to be fully developed. However, as can be anticipated from Fig.2, a plot similar to that of Fig.3 gives much larger values of $\Delta \sigma$ than those found for Tl2201. The slope found at the lowest $T$ for which $T / T_{0}$ is only 0.5 would extrapolate at low $T$ to a value $\alpha p>5$, which is absolutely not sound physically. We could obtain a similar conclusion for electron irradiated $\mathrm{YBCO}_{7}$ for which $p=1$ for the well known $T$ linear dependence of $\rho(T)$ 23]. Therefore all these data cannot be explained by weak $2 \mathrm{D}$ localization theory, as was also concluded for Zn substitution in $\mathrm{YBCO}_{6.7}$ [7].

Alternatively, we can wonder whether spin flip scattering effects can account for these large low $\mathrm{T}$ contributions. While for weak localization $\delta R_{2 D}$ scales with $R_{2 D}^{2}$ from (1), a Kondo like contribution to $R_{2 D}$ is expected to scale linearly with the defect content, that is with $R_{d}$, the increase of the residual resistivity. Indeed one can see in Fig. 4 that $\delta R_{2 D} / R_{d}$ follow the same $T$ dependence for a large range of $R_{d}$ values, if one omits to consider the dowturn associated with the proximity of the superconducting transition. Upward deviations with respect to this common behaviour begin to occur at low $T$ for $R_{d} \gtrsim 3.8 k \Omega$, that is $R_{2 D} \gtrsim 5 k \Omega$. This is even more prominent for the largest defect content in Fig. 1b which varies faster than $\ln T$, and might therefore correspond to the onset of strong localization.

It is interesting at this stage to compare these results with that obtained for underdoped Zn substituted samples [7]. In that case refined analyses, as done here, are impossible in the high $T$ range as the substituted sample has to be compared to a pure sample which is physically distinct. Systematic errors due to differences in the sample geometries and hole contents can then be hardly avoided. However superconductivity could be nearly suppressed with a $18 \mathrm{~T}$ field in $\mathrm{YBCO}_{6.7}$ with $2.7 \% \mathrm{Zn}$ [7] which allowed to evidence a $\ln T$ contribution to the resistivity (not to the conductivity) below $30 \mathrm{~K}$. This would be compatible with a Kondo contribution with a low $T_{K}$ value. A rough estimate for $\delta R_{2 D} / R_{d}$ yields a value for this $\ln T$ contribution about a factor two smaller than that obtained here. This order of magnitude agreement is quite satisfactory [24], as we are comparing here the influences of different microscopic defects.

In conclusion, we have evidenced that the low $T$ resistivity behaves quite differently in overdoped and underdoped systems. In the former case we have shown the relevance of weak localization theory, as $\Delta \sigma$ has a $\ln T$ variation independent of defect content and in rough quantitative agreement with the exponent of $R_{2 D}(T)$. A negative transverse magnetoresistance has been evidenced at low $T$, but further experiments are needed to determine the eventual contribution of interactions to the $\ln T$ term and the low $T$ variation of $\tau_{i}$.

We have shown that the large $\delta R_{2 D}$ measured in the underdoped case can be interpreted in terms of a Kondo scattering on the local moment induced by the "spin- less" defects. This implies per se that the scattering by such defects should saturate at low $T$ 25] and should only become unitary below $T_{K}$. Therefore, pure potential scattering by spinless impurities is only expected for $T<<T_{K}$. As large $T_{K}$ values, exceeding $200 \mathrm{~K}$, have been evidenced by NMR only for the overdoped case [6], pure elastic scattering is expected at low $T$ for these systems. This is then quite coherent with the occurence of weak localization contributions in the Tl2201 compound, if the NMR observations for Li impurities do apply as well for irradiation defects.

It is worth pointing here that $-\ln T$ contributions to $R_{2 D}$ were also observed for the "pure" underdoped LSCO [26] and $\mathrm{Bi}(\mathrm{SrLa}) \mathrm{CO} 2201$ [11]. They have been associated with a "peculiar" charge localization leading to a metal insulator transition (MIT) with decreasing hole content. It is usually assumed that some disorder is responsible for the anomalously low value of the optimum $T_{c}$ in these compounds and that this disorder might be at the origin of the $-\ln T$ term. Our data lead us to suggest that this unknown disorder might result primarily in a scattering on magnetic perturbations induced in the planes, as for spinless impurities. In both cases, one can wonder whether the scattering saturates at low $T$ or whether strong localization proceeds before this saturation can be detected. In other words, the MIT might result from the conjunction of uncontrolled disorder and of the enhanced sensitivity of the underdoped regime to this disorder. This would explain why the MIT is strongly dependent on the cuprate family.

We thank P. Lejay and A. Tyler for providing the single crystals and the technical staff of the LSI for their support during irradiation experiments.

(*) The irradiation experiments were performed at the LSI (Ecole Polytechnique, Palaiseau)

[1] T.R. Chien et al., Phys. Rev. Lett. 67, 2088 (1991)

[2] Y. Fukuzumi et al., Phys. Rev. lett.76, 684 (1996)

[3] S. Tolpygo et al., Phys. Rev. B53, 12454 and 12462(1996)

[4] F. Rullier-Albenque et al., Europhys. Lett. 50, 81 (2000)

[5] A.V. Mahajan et al., Phys. Rev. Lett. 72, 3100 (1994); Eur. Phys. J. B 13, 457 (2000)

[6] J. Bobroff et al., Phys. Rev. Lett. 83 , 4381 (1999)

[7] K. Segawa and Y. Ando, Phys. Rev. B, 59, R3948 (1999)

[8] K.Karpinska et al., Phys. Rev. Lett. 84, 155 (2000)

[9] G.J. Dolan and D.D. Osheroff, Phys. Rev. Lett. 43, 721 (1979)

[10] D.J. Bishop et al., Phys. Rev. Lett. 44, 1153 (1980)

[11] S. Ono et al., Phys. Rev. Lett. 85, 638 (2000)

[12] A.A. Abrikosov, Phys. Rev. B, 61, 7770 (2000)

[13] A. Legris et al. J. Phys. (France) I3, 1605 (1993)

[14] Y. Kubo et al, Phys. Rev. B 43, 7875 (1991)

[15] The fluences used for the determination of the relation 


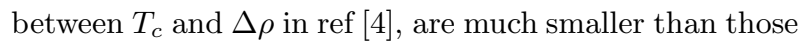
required in the present experiment.

[16] Let us point out that the fluence needed to visualize localization effects in this compound is three times larger than for YBCO6.6.

[17] P.A. Lee and T.V. Ramakrishnan, Rev. Mod. Phys.57, 287 (1985)

[18] D.J. Bishop et al., Phys. Rev. B 26, 773 (1982)

[19] G. Bergmann, Phys. Rev. B 28, 515 (1983)

[20] D. Abraham and R. Rosenbaum, Phys. Rev. B 27, 1413 (1983)

[21] R.S. Markiewicz and L.A. Harris, Phys; Rev. Lett. 46, 1149 (1981)

[22] A.I. Larkin, JETP Lett. 31,219 (1980). Interactions effects also give a $\ln T$ correction to $\sigma$ very similar to (11). Unfortunately we could not push the analysis farther on as the sample broke while mounting it to measure the longitudinal magnetoresistance.

[23] F. Rullier-Albenque et al., to be published

[24] Let us point out that the large applied field might even reduce the Kondo contribution in those experiments.

[25] Such a behaviour has been observed in Zn-substituted $\mathrm{La}_{1.85} \mathrm{Sr}_{0.15} \mathrm{CuO}_{4}$ [8].

[26] Y. Ando et al, Phys. Rev. Lett. 75,4662 (1995)

FIG. 1. The measured in plane resistivity $\rho$, scaled by the lattice constant $c$ and the number $n$ of $\mathrm{CuO}_{2}$ planes to give the $2 \mathrm{D}$ resistance $R_{2 D}=\rho n / c$, is plotted versus temperature for different irradiation conditions. The arrows indicate the sequence of measurements: increasing irradiation for straight arrows and long annealing at room $T$ for wiggled arrows. a) Tl2201 : the data down to $1.7 \mathrm{~K}$ has been taken in a different set up. b) $\mathrm{YBCO}_{6.6}$ : the extrapolated low $T$ variation of $\mathrm{R}_{2 D}^{0}(T)$ is shown as a full curve

FIG. 2. The low T increase $\delta R_{2 D}$ of the resistivity is plotted for $R_{2 D}$ of about $4.8 \mathrm{k} \Omega / \square$ for the two compounds. The different fitting procedures used to estimate $\delta R_{2 D}$ are described in the text: for $\mathrm{YBCO}_{6.6}$, fits with $a+b T^{p}$ or $a+b T+c T^{3}(\mathrm{a})$. For Tl2201, fit with $a+b T^{p}$ between $70 \mathrm{~K}$ and $150 \mathrm{~K}(\mathrm{~b})$ or between $150 \mathrm{~K}$ and $300 \mathrm{~K}$ (c).

FIG. 3. a) Plot of $\Delta \sigma$ versus $\ln T$ for different defect concentrations in Tl-2201. b) These curves are scaled by adjusting $T_{0}$ in equ. (酉).

FIG. 4. The low $T$ increase of $R_{2 D}$ is normalized to the defect contribution to the residual resistivity $R_{d}$ in $\mathrm{YBCO}_{6.6}$. Except for the onset of superconductivity, the data are found to collapse onto a unique curve as long as $R_{d} \lesssim 4 k \Omega / \square$. 


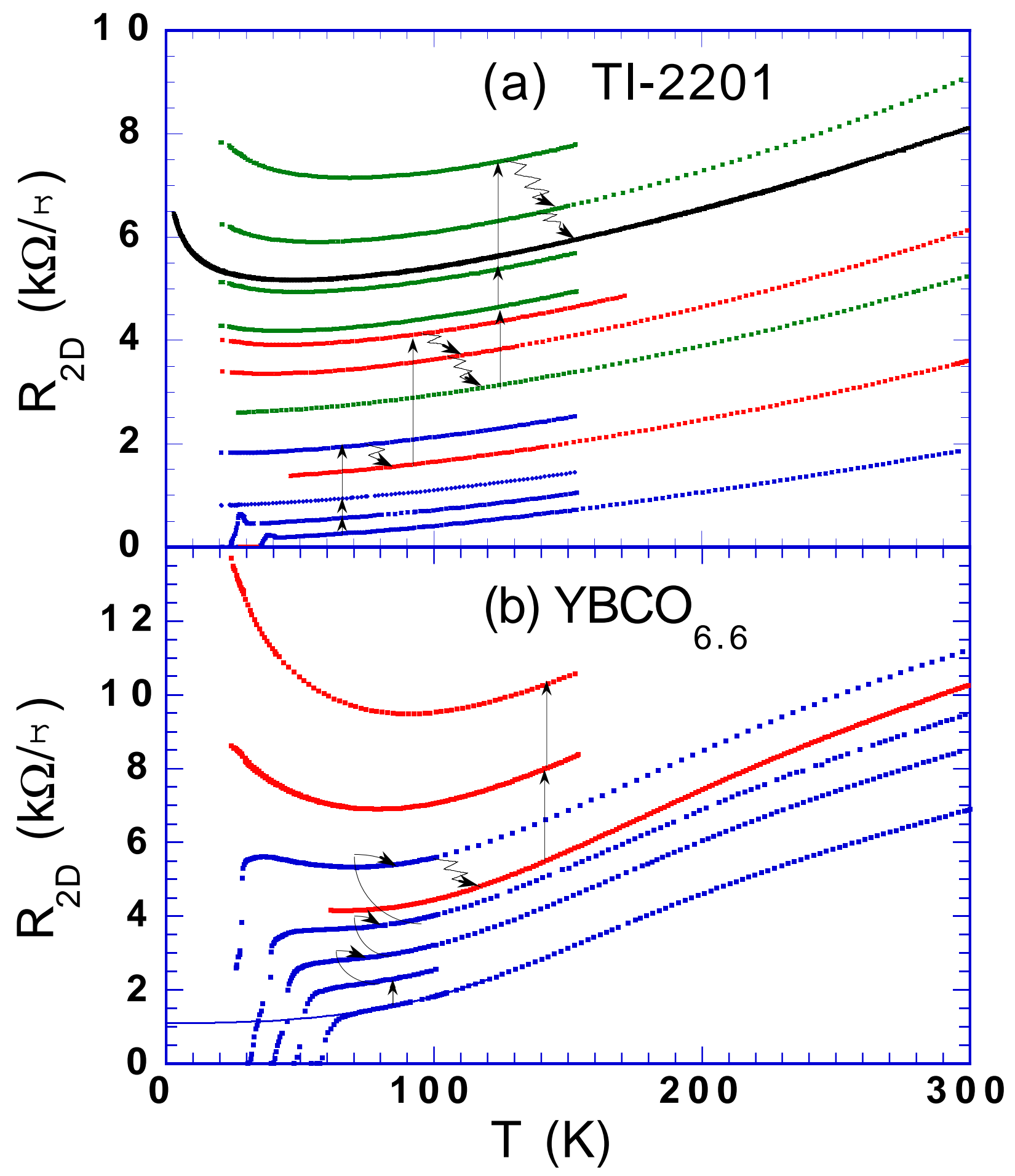

Fig.1 -F. Rullier-Albenque et al 


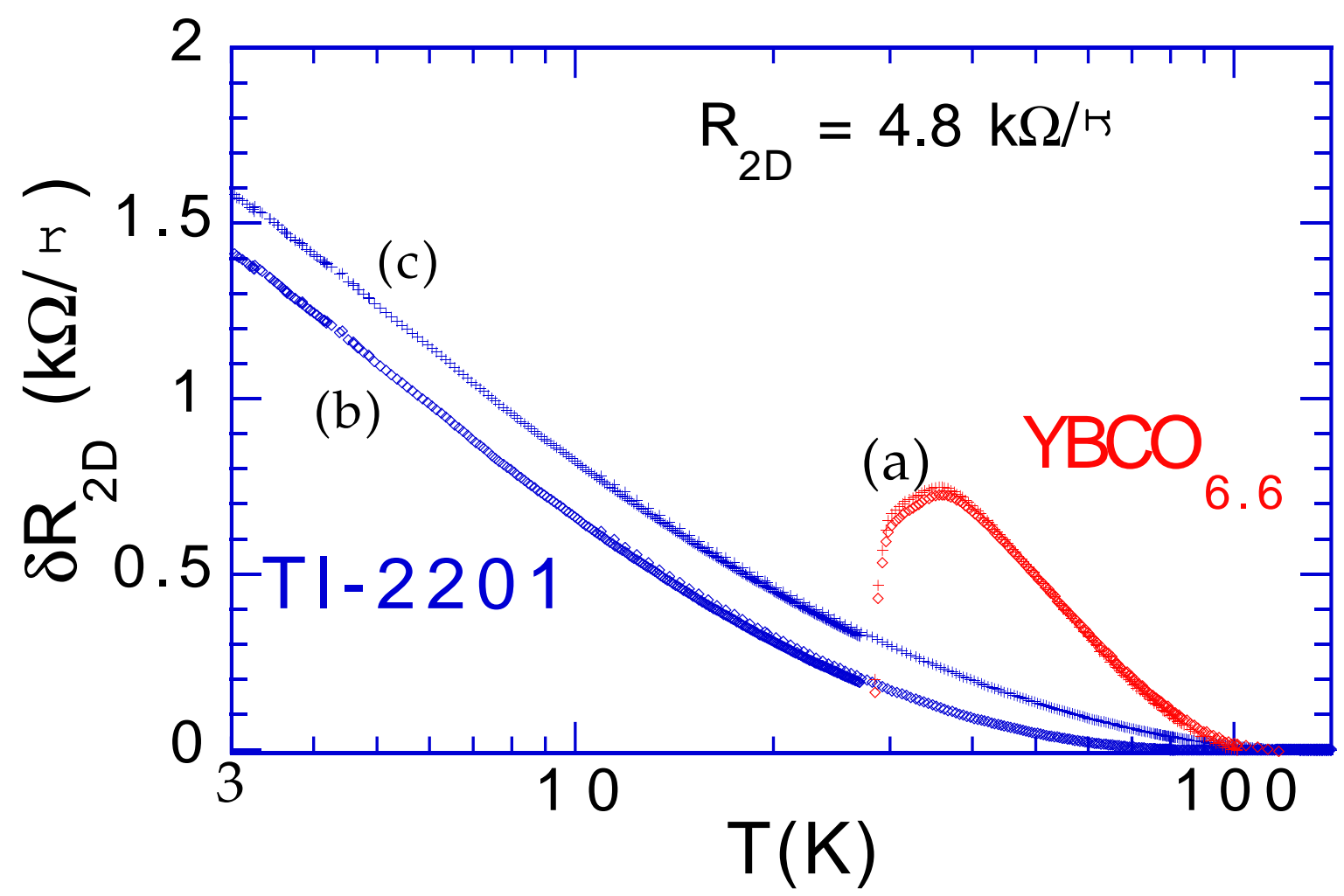

Fig.2 - F. Rullier-Albenque et al 


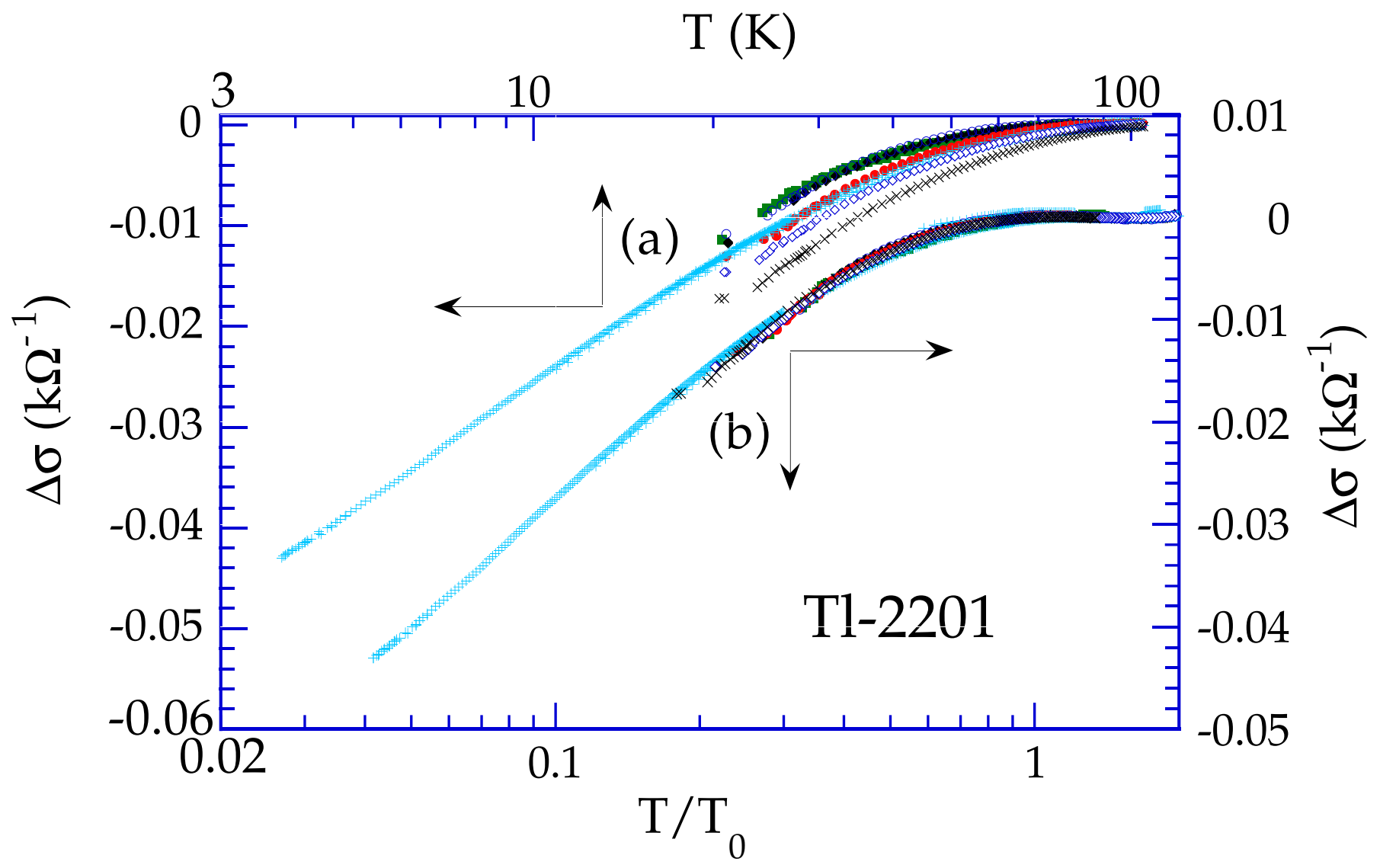

Fig.3 - F. Rullier-Albenque et al 


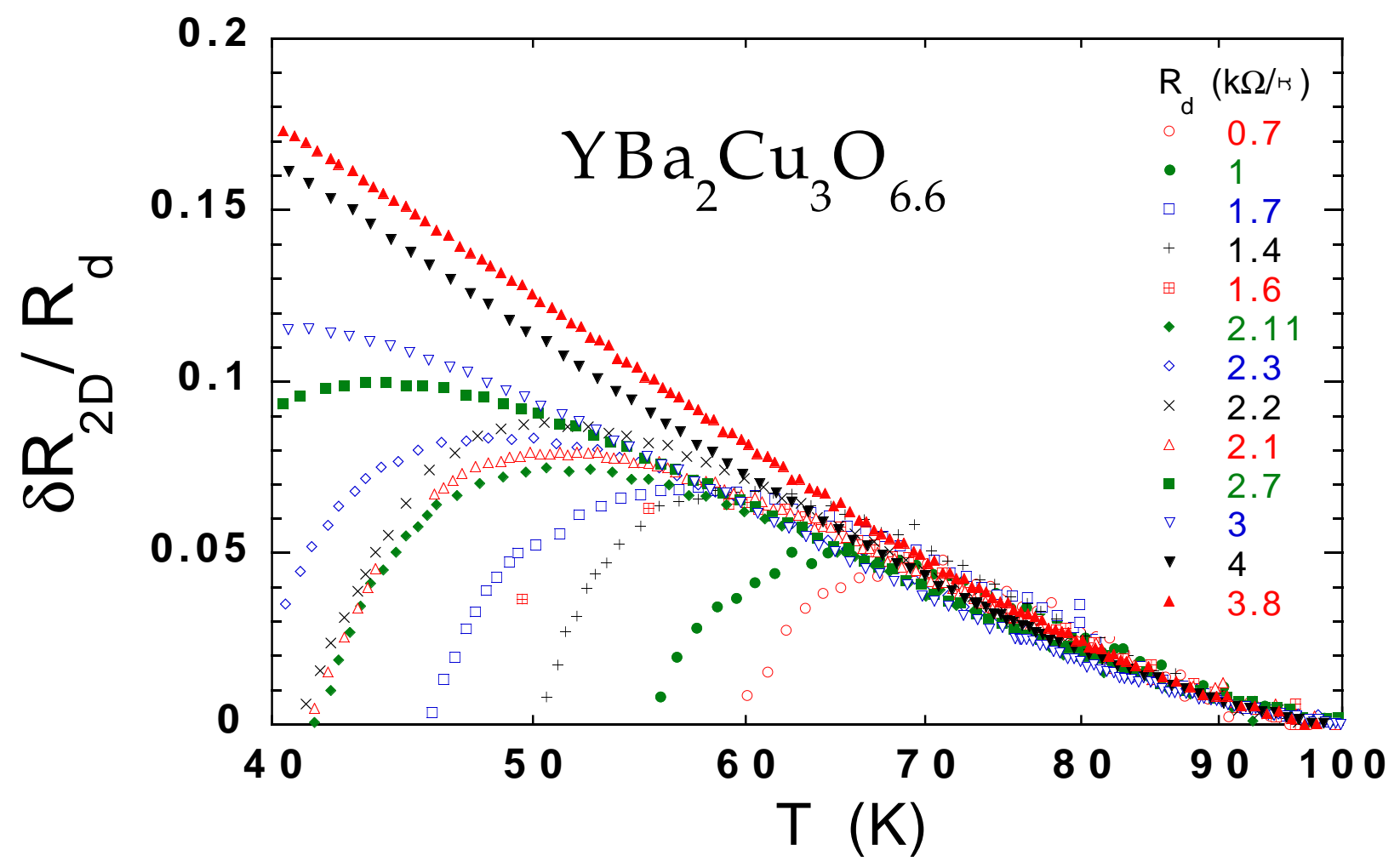

Fig.4 - F. Rullier-Albenque et al 